\title{
Studies on the effective physical parameters forchirp reduction in optical injection locked semiconductor lasers
}

\author{
S. N. Hosseinimotlagh ${ }^{1, ~}{ }^{\text {, }}$ M. Nikravesh ${ }^{2}$, H. Molaei ${ }^{3}$, M. Pezeshkian ${ }^{3}$ \\ ${ }^{1}$ Department of Physics, Colleges of Sciences, Islamic Azad University of Shiraz, Shiraz, Iran \\ ${ }^{2}$ Department of Physics, Science and Research Branch,Islamic Azad University,Fars,Iran \\ ${ }^{3}$ Department of Physics, Payam Noor University, Shiraz, Iran
}

Email address:

hoseinimotlagh@ hotmail.com (S. N. Hosseinimotlagh)

\section{To cite this article:}

S. N. Hosseinimotlagh, M. Nikravesh, H. Molaei, M. Pezeshkian. Studies on the Effective Physical Parameters for Chirp Reduction in Optical Injection Locked Semiconductor Lasers. American Journal of Physics and Applications. Vol. 2, No. 2, 2014, pp. 56-60. doi: 10.11648/j.ajpa.20140202.13

\begin{abstract}
In optical injection locking, the light from one laser (master laser)is injected into another laser slave laser. The injected light causes changes in the slave laser characteristics, and it can lock the slave laser lasing frequency to the ML lasingfrequency. The locking characteristics are determined by theamount of injected power and thefrequency difference between the master laser and slave laser.In this paper, the chirp of injection-locked semiconductor lasers has been theoretically investigated. First, chirpcharacteristics of semiconductor lasers are described. The chirp to power ratio has been calculated with including opticalinjection effect in lasers rate equation. Then, the effects of phase difference between master and slave laser and modulation frequency on chirp to power ratio are evaluated. The results of calculations show that the effect of phasedifference can be neglected, but optical injection, line width enhancement factor, and detuningfrequency have an effective role in chirp reduction of semiconductor lasers.
\end{abstract}

Keywords:Injection-Locked, Chirp, Semiconductor Lasers

\section{Introduction}

Injection locking of semiconductor lasers has been actively investigated recently. Theoretical calculations show that it is possible to increase laser bandwidth and to reduce noise and chirp with proper injection-locking conditions [1], [2].These properties can potentially lead to an increase of the transmission distance or bandwidth. The locking phenomenon occurs when an injection source laser (master laser) with a particular injection power is slightly detuned from the frequency of the injection locked laser (slave laser). Depending on the frequency detuning and the injection power, the injection-locked laser can be stably locked, unstably locked, or exhibit chaotic behavior. A DFB laser injection locked with another DFB was demonstrated to have three times the enhancement in the small signal modulation [3].

Thanks to its extremely low loss of the fiber light, the optical fibers have become a perfect tool for moving digital data in long distances. Intrinsic bandwidth of the fiber, in order of Tera Hertz, which offers great potential, only by the sender, receiver and indirect fibers are limited. Recently, not only the use of fiber optic digital, but analog and RF transmission is also taken into consideration. Light weight and low electromagnetic interference, optical fiber has become a ideal media for the remote antenna. Optical processing of radio signals also use in the optical domain. Directly modulated semiconductor lasers are compact and convenient sources for optical fiber systems.

Inlightinjection technique, we initially use two lasers that are not the same in wavelength. The light is injected from the first laser,called master laser, to another laser, called slave laser. The master laser and the slave laser's light combines and changes the internal field of the slave laser. Wavelength of the slave laser shifts to the wavelength of the master laser and the lasers in terms of both frequency and phase are locked together. The area that the wavelengths' of the lasers are locked together is called locked area. In this area the laser shows stable performance.

The technique of optical injection locking is frequently used to lock the frequency and stabilize the oscillation of a slave laser. The injection locking system is very simple, as shown in Fig. 1. 


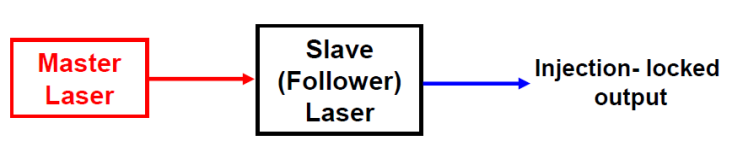

Fig 1.Optical injection system in semiconductor lasers.

Injection-locked semiconductor lasers are very useful for stabilizing the laser, however they sometimes shows a rich variety of dynamics. For optical injection locking, we prepare two lasers with almost the same oscillation frequencies and the frequency detuning between them must usually be within several $\mathrm{GHz}$. A light from a laser under a single mode oscillation (master laser) is fed into the active layer of the other laser (slave laser). Then, the two lasers synchronize witheach other in the same optical frequency under the appropriate conditions of the frequency detuning and the injection strength. The remarkable characteristics of optical injection locking in semiconductor lasers originated from the fact that the $\alpha$ parameter (line width enhancement factor) has a non-zero definite value, which makes semiconductor lasers very different from other lasers. As a viewpoint of laser dynamics, an optical injection from a different laser means the introduction of an extra degree of freedom to the semiconductor laser. Therefore, various dynamics are observed by optical injection, including stable and unstable injection locking, instabilities and chaos, and four-wave mixing depending on the locking conditions. Two important injection locking parameters are frequency detuning, $\Delta f$, which is the frequency difference between the master and the free-running slave lasers, and injection ratio, $R$, which is ratio between the injected power from the master laser and the lasing power of the free-running slave laser.

In fiber optic networks, simultaneously achieving high bit rate data transmission over long distances is very desirable, but due to the dispersion of optical fiber and optical electronics Chirp frequency component,it is not possible. Dispersion in optical systems, arises from the group velocity of light pulse's changes. That means that the single mode optical fibers, short wavelength components (Blue) are transferred faster than the long wavelength components (Red). The positive frequency part chirp light blue shift of the rising edge and the bottom edge of the red shift progressively flattened optical pulses and the pulse is observed at the receiver. One of the ways ofchirp reduction in semiconductor lasers, is optical injection technique by another semiconductor laser. An important advantage of optical injected lasers can be mention to minimize the chirp without complexity designed. Lasers can also follow main the same electronic circuits without high power consumption, to be launched.This greatly simplifies the circuit design, and cost transmitters modules at link digital decreases. Therefore Strong optical injection locking of semiconductor lasers can provide an excellent transmitter for high speed optical communication and a candidate for chaotic signals. The effects of optical injection locking mainly have two aspects: one is to improve the characteristics of the slave and the other is to synchronize the master and the slave.

In this paper, the chirp of injection-locked semiconductor lasers has been theoretically investigated.First, chirp characteristics of semiconductor lasers are described. The chirp to power ratio has been calculated with including optical injection effect in lasers rate equation. Then, the effects of phase difference between master and slave laser, thickness of active layer and detuning frequency on chirp to power ratio are evaluated.

\section{Theoretical Model}

The dynamics of injection-locked slave laser can be described by injection-locking rate equations. It was established by modifying the laser master equation within the framework of the semiconductor laser theory developed by Lamb [4] in 1964. For free-running lasers, based on the laser master equation, the laser field equation can be written as

$$
\frac{d E(t)}{d t}=\frac{1}{2}(G-a) E(t)+i \omega E(t)
$$

$E(t)$ is the laser field, $G$ is the gain from the active material inside the laser cavity, a is the loss including both material loss and mirror loss, which is equal to the inverse of the photon lifetime $1 / \tau \mathrm{p}$, and $\omega$ is the cavity resonance frequency. The laser field can be written in a complex form. The amplitude equation together with the carrier conservation equation for electrically injected diode lasers forms the well-known laser rate equations.

$$
\begin{aligned}
& \operatorname{If} E(t)=|A(t)| e^{i \varnothing(t)}, \text { then } \\
& \frac{d|A(t)|}{d t}=\frac{1}{2}(G-a)|A(t)| \\
& \frac{d n(t)}{d t}=\frac{J}{e d}-\frac{n(t)}{\tau_{s}}-G|A(t)|^{2}
\end{aligned}
$$

Where $n$ is the total carrier number in the active region, $J$ is the injected current, $e$ is the electron charge, and $\tau_{s}$ is the carrier lifetime. Notice that spontaneous emission term is not included here, which can become important if transient is considered, especially when the laser is being switched on and off.

The first thorough theoretical study on OIL of semiconductor lasers was done by Roy Lang [5] in 1982. By adding in the external light injection term, the master equation changes to

$$
\frac{d E_{s}(t)}{d t}=\frac{1}{2}\left[G(n)-\frac{1}{\tau p h}\right] E_{s}(t)+i \omega(n) E_{s}(t)+k E_{i n j}(t)
$$

Where $\quad E_{s}(t)=A(t) e^{i\left[\omega_{f r}+\phi_{s}(t)\right]} \quad$ and $\quad E_{\text {inj }}=$ $A(t) e^{i\left[\omega_{i n j}+\phi_{i n j}(t)\right]}$ are the complex fields of the slave and the master laser. In addition, as the external field enhances the stimulated emission inside the slave laser cavity, which will reduce the carrier number $N$, the index of refraction, hence the cavity resonance will be red shifted. This is represented by $\omega$ as a function of $N$ in Equation (4). 
Plugging the complex form of both the injection field and the slave laser field into the modified master equation and separating the real and imaginary parts using the master laser phase as the reference, a set of three equations can be derived and they are the well- known rate equations for an injection-locked laser [6].

$$
\begin{aligned}
& \frac{d A(t)}{d t}=\frac{1}{2} G\left(n(t)-n_{t h}\right)\left(1-\varepsilon_{s}|A|^{2}\right) A(t)+k_{i n j} A_{i n j} \cos \phi(t) \\
& \frac{d \phi(t)}{d t}=\frac{\alpha}{2} G\left(n(t)-n_{t h}\right)\left(1-\varepsilon_{s}|A|^{2}\right)-k_{i n j} \frac{A_{i n j}}{A(t)} \sin \phi(t)-\Delta \omega_{i n j} \\
& \frac{d n(t)}{d t}=\frac{J}{e d}-\gamma_{N} N(t)-\left\{\gamma_{P}+G\left(n(t)-n_{t h}\right)\right\}\left(1-\varepsilon_{s}|A|^{2}\right) A(t)^{2}
\end{aligned}
$$

where $E(t)$ is the normalized the field and $A^{2}(t)=S$ the total number of photons, $n_{\mathrm{th}}$ is the threshold carrier number, $\Delta \omega$ $=\omega_{\text {inj }}-\omega_{\text {fr }}$ is the frequency difference between the master and the slave laser, often refer to as the frequency detuning, $\varphi(t)=\varphi_{\mathrm{s}}(t)-\Delta \omega t-\varphi_{\text {inj }}(t)$ is the relative phase between the master and the slave laser field, $\alpha$ is the line width enhancement factor of the slave laser, $\kappa=\left(v_{\mathrm{g}} / 2 L\right)(1-R)^{1 / 2}$ is the coupling coefficient of the master light into the slave laser depending on the group velocity $v \mathrm{~g}$, the slave laser cavity length $L$ and its mirror reflectivity $R$, and finally $G$ is the gain coefficient.

Since frequency chirping exhibits universally for all kinds of semiconductor lasers and severely limits the transmission distance of direct-modulated laser, studies on the origin of chirp dated back to the mid-80s [7-9]. This can be understood by the schematic illustration in Figure 2. If the current of a diode laser is modulated by a certain data pattern, the carrier density, which is pumped up by the current, follows the same pattern accordingly. Therefore, the light intensity generated by carrier radiative recombination will also follow the data pattern. However, due to the line width enhancement factor of the semiconductor material, the refractive index is coupled to the carrier density, hence the carrier density modulation will result in the index modulation which eventually leads to the residual frequency modulation known as chirp.

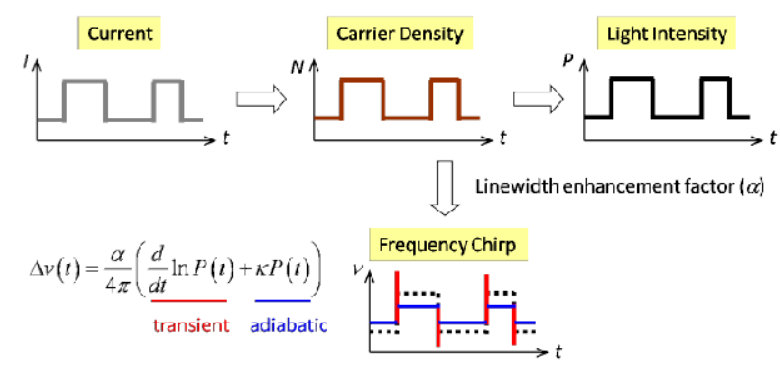

Figure 2.Frequency chirping of semiconductor lasers. Positive chirp exhibits for all types of semiconductor lasers due to the existence of line width enhancement factor.

A thorough study [10] on semiconductor laser dynamics including the nonlinear gain effect shows that chirp consists of two terms

$$
\Delta v(t)=\frac{\alpha}{4 \pi}\left(\frac{d}{d t} \ln P(t)+k P(t)\right)
$$

Where $\Delta v$ is frequency chirp, $P$ is laser power, $\kappa$ is the a function of the gain compression factor [11]. The first term in Eqn (8) represents the transient chirp, which is associated with the fast rising and falling edge of the signal, while the second term represents the adiabatic chirp, which is caused by the nonlinear gain effects, such as carrier heating and spectral hole burning, and determined by the absolute power level.In semiconductor lasers chirp most important criterion, which can be applied to the frequency deviation chirp, the deviation can be defined:

$$
C P R=\left|S \frac{\Delta \phi}{\Delta A}\right|=\frac{\alpha}{2 A_{0}}\left|S \frac{S+\omega_{A}}{S+\omega_{B}}\right|
$$

with

$$
\omega_{B}=Z\left[\cos \phi_{0}-\alpha \sin \phi_{0}\right] \text { and } \omega_{A}=Z\left[\cos \phi_{0}+\sin \phi_{0} / \alpha\right]
$$

This equation is the fact that the phase- dependent modulation CPR completely determines the response .The above equation implies that these are thread -locked semiconductor lasers by optical injection, reduce frequency or kill chirp to improve and optimize the performance of the laser is used .chirp of the semiconductor laser can be locked to an optical injection locking the stable area modulated as a function of frequency in Figure 3 is shown for three different injection ratios .As can be seen from the increase of injection, chirp frequency decreases.

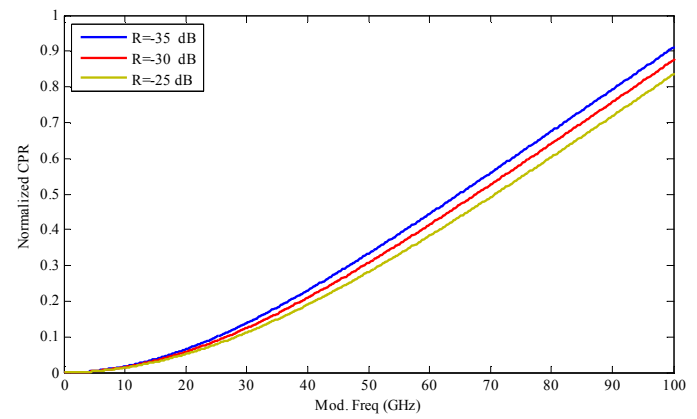

Figure 3.Frequency chirp from locked semiconductor lasers with optical injection at different injection rates. 
Since the line width enhancement factor $\alpha$ directly affects the frequency chirp of these lasers, and it is also a contributing factor (the change in refractive index is achieved by optical injection), so the effect of these factors is essential in reducing the frequency chirp.In Figure 4 the effect of this factor is plotted for a constant injection rate.This figure represents the reduction factor increases the width of the line, has a significant impact in decreasing the frequency chirp.

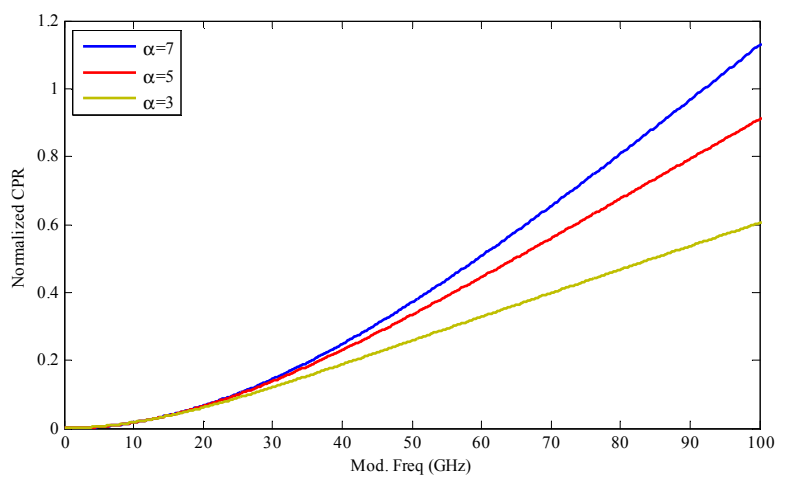

Figure 4. Frequency chirp from locked semiconductor lasers with optical injection at different linewidth enhancement factor.

However, using the fact that the master and slave lasers and can have different phase, we will initially look on the effect of phase difference on the master and slave lasers on frequency chirp .Figure 5 shows the effect of phase on the chirp. The modulation frequency is usually above $10 \mathrm{GHz}$, we find from Figure 5 that the initial phase difference between the two laser frequencies do not effect on the chirp, so you can be regardless of the effect on chirp .Therefore, in the calculation of chirp we consider the initial phase difference zero .

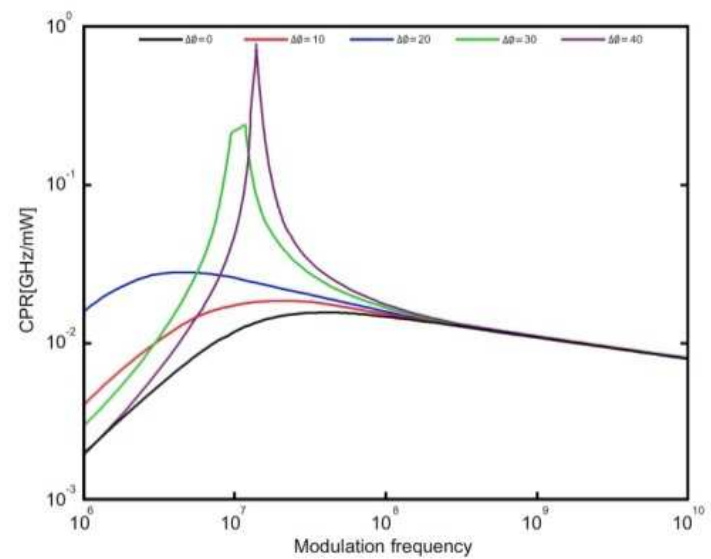

Figure 5. Frequency chirp via modulation frequency in varies initial phase depending on the frequency of injecting $100 \mathrm{~dB}$.

As Figure 6 shows the master laser power cuts,CPR because of the dominance of non-injected mode and spontaneous emission, nonlinear effects appear. At high injection rates, the effects of the nonlinear dependence of $\mathrm{CPR}$ is reduced and the frequency dependent.Chirp will be linear relationship at high frequencies, and the frequency is independent of the injection ratio, because the frequency of the modulation frequency of the spontaneous emission is negligible.

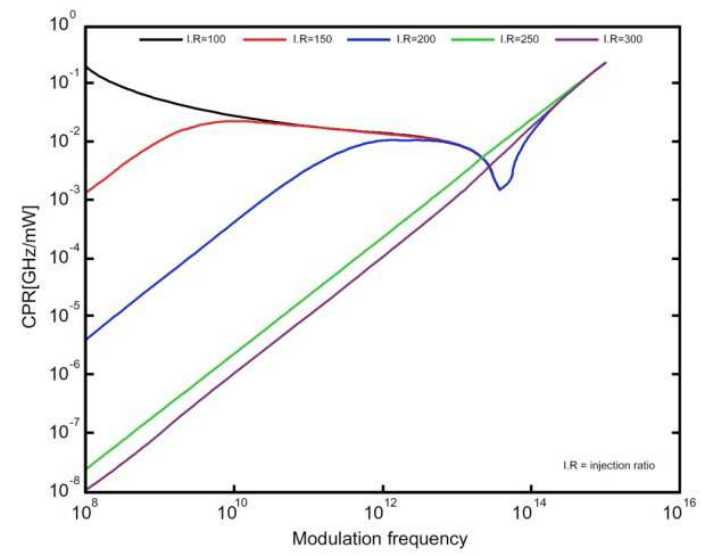

Figure 6. Chirp to be based on frequency modulation ratio for different injection rates.

\section{Conclusions}

In this paper, chirp of semiconductor lasers were investigated.To do this, first with the help of optical injection locking of semiconductor laser rate equations, we can obtain the chirp to power ratio, Then, the effects of phase difference between master and slave laser, injection ratio and modulation frequency on chirp to power ratio are evaluated.The results of calculations show that the effect of phasedifference can be neglected, but optical injection, injection ratio andline width enhancement factor, have an effective role in chirp reduction of semiconductor lasers.

\section{References}

[1] J. M. Liu, H. F. Chen, X. J. Meng, and T. B. Simpson, "Modulation bandwidth, noise, and stability of a semiconductor laser subject to strong injection locking," IEEE Photon. Technol. Lett., vol. 9, pp. 1325-1327,Oct. 1997.

[2] V. Annovazzi-Lodi, A. Scire, M. Sorel, and S. Donati, "Dynamic behavior and locking of a semiconductor laser subjected to external injection," IEEE J. Quantum Electron., vol. 34, pp. 2350-2357, Dec. 1998.

[3] X. Meng, T. Chau, and M. C. Wu, "Experimental demonstration of modulation bandwidth enhancement in distributed feedback lasers with external light injection," Electron. Lett., vol. 34, pp. 2031-2032, Oct. 1998.

[4] W. E. Lamb, "Theory of an optical maser," Phys. Rev., 134(6A), 1429-1450, 1964.

[5] R. Lang, "Injection locking properties of a semiconductor laser,” IEEE J. Quantum Electron., QE-18,976-83, 1982.

[6] A. Murakami, K. Kawashima, and K. Atsuki, "Cavity resonance shift and bandwidth enhancement insemiconductor lasers with strong light injection," IEEE J. Quantum Electron.39, 1196-204, 2003. 
[7] T. L. Koch, \&J. E.Bowers, Nature of wavelength chirping in directly modulated semiconductorlasers. Electron.Lett. 20, 1038-1040, 1984

[8] F.Koyama, \&Y.Suematsu, Analysis of dynamic spectral width of dynamic-single-mode (DSM)lasers and related transmission bandwidth of single-mode fibers. IEEE J. Quantum Electron. QE-21, 292-297, 1985

[9] R. A. Linke, Modulation induced transient chirping in single frequency lasers. IEEE J. QuantumElectron. QE-21, 593597,1985

[10] T. L. Koch and R. A. Linke, "Effect of nonlinear gain reduction on semiconductor laser wavelengthchirping" Appl. Phys. Lett. 48, 613-615, 1986

[11] R. S. Tucker, "High-speed modulation of semiconductor lasers," J. Lightwave Technol. 3, 1180-1192,1985. 